\title{
¿Por qué México es un país altamente vulnerable al cambio climático?
}

\author{
Víctor Manuel Rodríguez-Moreno ${ }^{1 \S}$ \\ Guillermo Medina-García ${ }^{2}$ \\ Gabriel Díaz Padilla ${ }^{3}$ \\ José Ariel-Ruiz Corral ${ }^{4}$ \\ Juan Estrada-Avalos ${ }^{5}$ \\ Jorge Ernesto Mauricio Ruvalcaba ${ }^{1}$
}

${ }^{1}$ Campo Experimental Pabellón-INIFAP. Carretera Aguascalientes-Zacatecas km 35.5, Pabellón de Arteaga, Aguascalientes. CP. 20668. (jorge.ernesto.mauricio@gmail.com). ${ }^{2}$ Campo Experimental Zacatecas. Carretera Zacatecas-Fresnillo km 24.5, Calera de Victor Rosales, Zacatecas. CP. 98500. (medina.guillermo@inifap.gob.mx). ${ }^{3}$ Campo Experimental Cotaxtla. Carretera Xalapa-Veracruz km 3.5, Ánimas, Xalapa, Veracruz. CP. 91190. (diaz.gabriel@inifap.gob.mx). ${ }^{4}$ Universidad de GuadalajaraDepartamento de Ciencias Ambientales. Camino Ing. Ramón Padilla Sánchez núm. 2100, La Venta del Astillero, Zapopan, Jalisco. CP. 45110. (ariel.ruiz@ academicos.udg.mx). ${ }^{5}$ Centro Nacional de Investigación Disciplinaria-RASPA. Margen Derecha Canal Sacramento km 6.5, Gómez Palacio, Durango. CP. 27130. (estrada.juan@inifap.gob.mx).

${ }^{\S}$ Autor para correspondencia: rodriguez.victor@inifap.gob.mx.

\section{Resumen}

Este manuscrito proporciona una visión general sobre la vulnerabilidad de México, como región geográfica, a los impactos del cambio climático. Es en el dinamismo del sector primario donde los cambios climáticos globales climáticos globales tienen mayor influencia. Para reescalar las observaciones globales a escala local, regional y nacional, el INIFAP como centro público de investigación, aporta soluciones tecnológicas al alcance de los usuarios, tomadores de decisiones, investigadores, académicos y consultores. Desde su creación, el instituto mantiene líneas de investigación para estudiar los impactos del cambio climático en lo agrícola, pecuario y forestal. Esta la lleva a cabo a través de la organización de encuentros nacionales e internacionales de intercambio técnico-científico y la ejecución de proyectos de investigación y de servicios. A través de la integración y análisis de bases de datos, de la implementación de técnicas de máquinas de aprendizaje en arquitecturas de cómputo, series de tiempo de imágenes satelitales y datos de procesos de intercambio entre la cubierta del suelo y la atmósfera, el Laboratorio Nacional de Modelaje y Sensores Remotos oferta al usuario productos y servicios basados en TICs. Pronósticos numéricos de lluvia y sequía, conforman la oferta institucional para proveer de información útil para la mitigar los efectos del cambio climático en el sector primario. A través de estos productos y servicios se promueve un cambio de paradigma para el estudio de los impactos del cambio climático en México.

Palabras clave: cambio climático, México, sector primario, variabilidad climática.

Recibido: febrero de 2021

Aceptado: mayo de 2021 
México es el catorceavo país más extenso en el mundo, el onceavo en número de habitantes y el séptimo en producción de petróleo. Por su geografía, las zonas costeras serían las más afectadas por aumentos en la temperatura del aire, los eventos extremos como sequía y ciclones, deslizamientos de tierra, y disminuciones del volumen de precipitación. Estos impactos generan nuevos riesgos para la población e inestabilidad a los ecosistemas (Oswald-Spring y Brauch 2009b). Las variables asociadas a la expresión del clima son los indicadores que 'miden' el impacto del cambio climático. Pronosticar su tendencia e intensidad en el corto, mediano y largo plazo evidencian su estacionalidad y su estrecha relación con la disponibilidad del recurso agua. El Instituto Nacional de Investigaciones Forestales, Agrícolas y Pecuarias (INIFAP) ha mantenido líneas de investigación para estudiar en paralelo la evolución de los indicadores del impacto del cambio climático y su impacto en los sistemas de producción agrícola, pecuario y forestal. A través de paquetes tecnológicos específicos por cultivo y región geográfica y el desarrollo de servicios web, ha mantenido el interés entre la comunidad científica por estudiar el cambio climático global y los métodos de reescalamiento de soluciones globales a escala local, regional y nacional.

En esta tarea de explorar y analizar los efectos del cambio climático, el concepto de los grandes números toma relevancia. El crecimiento exponencial de las grandes bases de datos que dan seguimiento a la dinámica de los ciclos biogeoquímicos del planeta, demanda disponer de recursos humanos entrenados, alta capacidad de cómputo y de almacenamiento de datos. En la ejecución de los procesos de cómputo en la nube, la selección de herramientas de análisis de grandes bases de datos, las plataformas de análisis inteligente y las soluciones tecnológicas, juegan un papel relevante (Almgren et al., 2019; Oury y Sing, 2018). Se pretende, por un lado, comprender el dinamismo evolutivo de los ciclos biogeoquímicos del planeta, como el ciclo del agua, el ciclo del carbono y los movimientos de rotación, traslación y por otro lado, derivar soluciones en las que sustentar programas específicos de prácticas de mitigación a los efectos del cambio climático.

Entre los principales impactos, las variaciones en la producción agrícola -que no es alternancia en la producción- se observan fuertemente asociadas a un componente geográfico. Cambios en la geografía de la producción disminución del recurso hídrico, baja en el rendimiento de cultivos y el aumento en la frecuencia e intensidad de eventos meteorológicos extremos asociados a temperatura del aire y lluvia, y que resultan en perturbaciones a los ecosistemas (Fellmann, 2012).

Después de casi medio siglo de desarrollo y diseño técnico, se han puesto en operación sistemas 3D de observación de los sistemas regentes en la Tierra. Registran el espectro electromagnético en las regiones de luz visible, infrarroja y de microondas. Con anterioridad a estos sistemas, el registro de datos se hacía a escala local y raramente a escala regional. Se utilizaban sensores interconectados en red. El INIFAP actualmente administra una base de datos clave de 340 estaciones meteorológicas automáticas que registran cada $15 \mathrm{~min}$ paquetes de datos de seis variables: temperatura del aire $\left({ }^{\circ} \mathrm{C}\right)$, humedad relativa $(\%)$, lluvia acumulada $(\mathrm{mm})$, radiación solar $\left(\mathrm{W} \mathrm{m}^{-2}\right)$, dirección y velocidad del viento $\left(\mathrm{m} \mathrm{s}^{-1}\right)$.

Sin embargo, la representatividad espacial del punto de monitoreo se restringe solo a las variables registradas, pasando desapercibidos otros fenómenos geofísicos y bióticos por la insuficiente densificación de los dispositivos en el terreno y la poca resolución vertical de los datos (Hua-Dong et al. 2015). En paralelo al diseño de los sistemas de observación, las técnicas de análisis de datos han sido convertidas a arquitecturas de datos, con alta capacidad de manejo y almacenamiento. 
En la complejidad de estudiar el cambio climático, las grandes bases de datos son el recurso necesario para dar seguimiento a los cambios estacionales (Manogaran et al., 2018), entender el paradigma del cambio (Faghmous et al., 2014), identificar los riesgos (Ford et al., 2016) y explorar las fuentes de datos blandos. El arreglo de datos en series de tiempo multitemporales, multiorigen, multivariable y multiescala, hace necesario diseñar arquitecturas de cómputo y utilizar herramientas de administración de grandes bases de datos.

Las técnicas de máquinas de aprendizaje (MA) se distinguen por su capacidad predictiva y de administración de grandes bases de datos. A través de su visión de cómputo, pueden administrar dominios como sistemas de distribución de electricidad, manejo del agua potable en ciudades (Hao, 2019), unidades de producción agrícola, pecuaria y forestal, y simular el clima en el corto, mediano y largo plazo. El dominio está en función de la solución de reescalamiento. Por lo general, un aumento en la resolución espacial reduce la cobertura en el terreno.

Los clasificadores de primera línea se usan en insuficiencia de datos, bajo poder de cómputo y reducida oferta de algoritmos de búsqueda. Son métodos de análisis parsimoniosos y de baja complejidad como bosques aleatorios, máquinas de soporte vectorial y redes neuronales.

El presente manuscrito aborda el uso de técnicas de análisis de datos para producir indicadores numéricos sobre la vulnerabilidad de México al cambio climático. Se hace énfasis a como a través del análisis de grandes bases de datos y técnicas de máquinas de aprendizaje, se obtienen indicadores pronosticados a escala local, regional y nacional que coadyuvan al diseño de programas estratégicos de mitigación al cambio climático. Asimismo, delinea el aporte del INIFAP como centro público de investigación (CPI) en el estudio del cambio climático. Es conveniente destacar que la revisión no aborda la diversidad de conceptos e interpretaciones sobre vulnerabilidad, ni sobre el alcance de los métodos de análisis utilizados.

\section{Descripción y alcances del problema}

El cambio climático en México tiene componentes sociales y económicos que son evidentes a escala local, regional y nacional. Las afectaciones son palpables por la tasa de cambio del uso del suelo, la calidad del aire, el desabasto de agua y la deficiente proveeduría de servicios esenciales a la población. El espacio geográfico de México representa una mega diversidad (Groombridge y Jenkin, 2002), con extensión territorial de 1959 millones de $\mathrm{km}^{2}$ en la plataforma continental, donde dos terceras partes son consideradas árida o semiárida (50-100 mm de lluvia acumulada anual) y una tercera parte húmeda (lluvia acumulada anual $>2000 \mathrm{~mm}$ ).

La variabilidad climática es alta y la expresión climática está fuertemente influenciada por factores asociados a la topografía del terreno y a la temperatura superficial del mar. Prevalecen condiciones cálidas y secas en la región Noroccidental en el Desierto sonorense, donde la precipitación anual es de apenas $100 \mathrm{~mm}$, hasta los climas tropicales húmedos de Chiapas y la región costera del Golfo de México donde la lluvia anual acumulada es mayor a 2000 mm (Lieverman, 1999).

La expresión climática tiene su origen en cinturones latitudinales de circulación atmosférica. Los vientos del oeste acarrean precipitaciones al norte de México en invierno. Cuando los vientos entregan lluvias en verano en las regiones centro y sur del país, es por condiciones estables y secas, que se extienden hasta la zona de convergencia intertropical (ZCIT). 
Otras influencias clave en la precipitación son los huracanes de otoño en las costas del Caribe y el Pacífico, los monzones de verano en el norte y la alta presión de verano que interrumpe el flujo de aire húmedo y crea un período de condiciones secas conocido como canícula. La topografía montañosa y variante de México domina muchas otras influencias climáticas que generan temperaturas más frías y mayores precipitaciones en las tierras altas y en la Mesa central y lluvias ligeras detrás de las montañas costeras (Metcalfe, 1987).

El espacio geográfico puede ser representado por siete grandes conceptos: lugar, espacio, ambiente, interconectividad, sustentabilidad, escala y cambio (Groombridge y Jenkin, 2002). El concepto de lugar se ajusta según la relevancia del ecosistema por sus características intrínsecas. Espacio, es el cómo y el porqué del acomodo de los elementos del ecosistema según las necesidades de la población. El concepto de ambiente se relaciona según su influencia en el hábitat, en las formas de vida y sus interrelaciones. Interconectividad es la interrelación entre las cosas vivas y no vivas, la idea central es que un evento que ocurre en un lugar puede generar un cambio en otro lugar y que ningún elemento geográfico puede estudiarse por separado.

En la práctica, sustentabilidad es una interconectividad de elementos como: salud ambiental, igualdad social, explotación racional del capital natural y el fortalecimiento de las cadenas de producción. Escala es la manera en que los eventos geográficos y las características de origen pueden ser explicadas a diferentes niveles, puede ser local (distribución de agua en el suelo), regional (onda de calor), nacional (frentes fríos, onda polar, trayectoria de un huracán, etc.). El concepto de cambio analiza como los ecosistemas y las formas de vida que constituyen el capital natural, han cambiado en un periodo de tiempo.

En México, el número de servicios ambientales asociados a los sistemas de producción es un punto distintivo por su evidente variación geográfica. Por mencionar algunos, calidad del aire, cosecha de agua, calidad del agua, uso sustentable del suelo, diversidad biológica, conservación de cadenas tróficas y fortalecimiento de las cadenas de producción de alimentos.

Las prácticas agrícolas de los sistemas de producción son geográficamente dependientes. Se clasifican según el enfoque de la producción en empresarial y social. Las prácticas empresariales son implementadas con sentido de mercado, donde el destino de la producción son mercados de consumo de exportación. En conjunto representan los indicadores agrícolas más relevantes como: mayor volumen de producción, rendimiento de cultivos y diversificación del mapa de cultivos, incluyen mayormente explotaciones perennes como guayaba, cítricos, nogal y manzano.

En las prácticas agrícolas con enfoque social, la producción es mayormente para mercados de consumo nacional o para autoconsumo. Su aporte a los indicadores económicos agrícolas es menor que los de la práctica empresarial, la productividad está condicionada al ciclo de lluvias.

Por efectos del cambio climático, los impactos económicos y sociales en el sector primario, serán cada vez más graves conforme se agudicen la disponibilidad del recurso agua y la variabilidad climática. Algunos de los efectos evidentes son: variaciones en los ciclos biológicos de organismos plaga (vertebrados, hongos, fitoplasmas y bacterias), condición de sequía, calidad del aire (volumen de material particulado y presencia de contaminantes como dióxido de carbono, dióxido de azufre y compuestos nitrogenados), ocurrencia de fenómenos meteorológicos extremos (granizada, chubasco, huracán y onda de calor), incendios forestales, y abatimiento del manto freático. 
Otro tipo de impactos, son los asociados a las condiciones meteorológicas. Se propone agruparlos en tres categorías: los asociados a la temperatura del aire, los asociados al ciclo de lluvias y los asociados a las prácticas sustentables. Los asociados a la temperatura del aire son: por ejemplo, condición de sequía, onda de calor, acumulación de unidades calor y de unidades frío, duración de la estación de crecimiento de los cultivos y tasa de evapotranspiración. Los asociados al ciclo de lluvias identifican las regiones geográficas por el volumen de lluvia recibido, la discrepancia está en el tiempo que ocurre y en el periodo de retorno. Los asociados a las prácticas sustentables tienen aplicación sobre el manejo del capital natural, agricultura de conservación, mejoramiento de recursos genéticos, agricultura de precisión, obras de infraestructura hidráulica y cosecha de agua.

\section{El cambio climático como política de estado}

En su visión futurista para 2025, la organización Tierra Futura identificó siete retos por enfrentar: sinergia y comercio de agua, energía, producción de alimentos, descarbonización de los sistemas socioeconómicos, avalúo y gobernanza de biodiversidad (Bauer et al., 2012), funcionamiento del ecosistema y servicios ambientales y resiliencia social en ruta hacia el desarrollo sustentable. orientados al espacio geográfico de México, se esquematizan en la Figura 1.

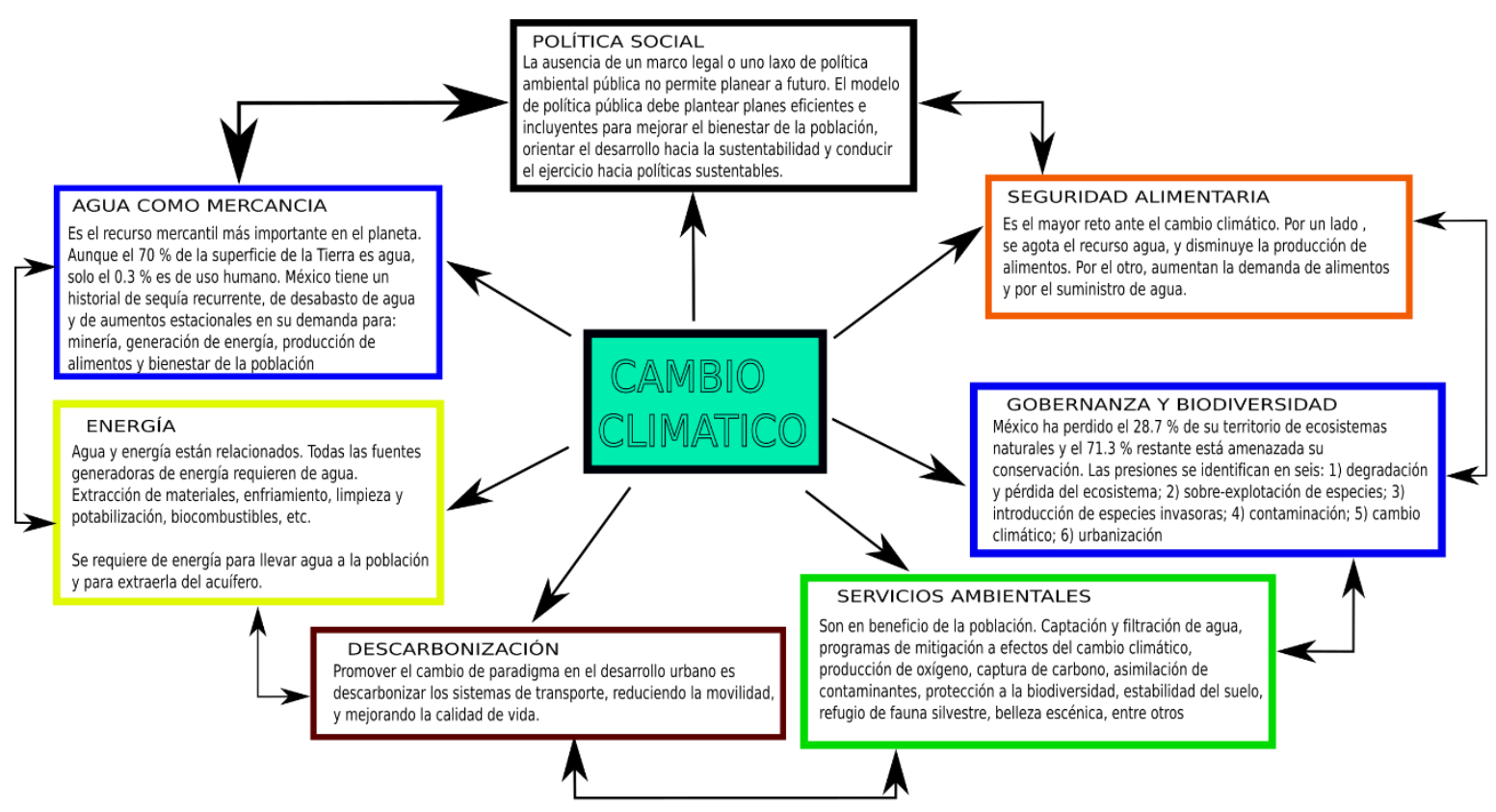

Figura 1. Interrelaciones del cambio climático como política de estado.

De la Figura1, se destaca que las energías renovables tienen un desarrollo potencial regionalizado: al norte se promueve la generación de energía solar, al centro y noroeste la geotérmica y al sur la energía eólica (impacto social consultores, 2021). En cuanto a seguridad alimentaria, México es el cuarto productor de maíz en el mundo, pero también es un importante importador y consumidor. La demanda de maíz blanco en el mercado de consumo interno está cubierta, pero el país es deficitario en la producción de maíz amarillo. La escasez se cubre con importaciones de Estados Unidos de América, Brasil y Argentina. El volumen de maíz importado se observa con tendencia alcista, con una tasa media de crecimiento anual de 5.8\% (CIMA, 2020). Resaltar que el grano de 
maíz es clave para la seguridad alimentaria y que la baja en el rendimiento histórico en los principales estados productores está relacionada con eventos meteorológicos extremos como heladas, granizadas, desabasto de agua, y huracanes.

México es un país dependiente de los combustibles fósiles, cuya producción y oferta energética se cubren mayoritariamente con gas y el petróleo. El más reciente balance energético arrojó que $85 \%$ de la oferta interna bruta fue cubierta por hidrocarburos que, durante 2018, disminuyeron 1.36 puntos porcentuales a su participación. El gas natural y los condensados aportaron $47 \%$ de la oferta total, seguido del petróleo y los petrolíferos con 38\% (SENER, 2019)

Se estima que en algún momento del periodo 2040-2050 el petróleo será desplazado por completo por las energías alternativas, sobre todo las renovables y limpias, con lo que su uso energético se reduciría de forma significativa (Senado de la República, 2003; Del Río et al., 2016; Cherif et al., 2017). Dos factores que incentivan la innovación tecnológica podrían dar lugar a la consolidación de la tercera revolución energética: el costo energético y económico de extraer petróleo y las presiones internacionales para reducir las emisiones de gases de efecto invernadero.

La Política Nacional de Cambio Climático de México se concibe como el conjunto de intervenciones públicas desarrolladas por los tres órdenes de gobierno que contribuyen a reducir las emisiones de gases y compuestos de efecto invernadero y transitar hacia una economía baja en carbono, así como también, a disminuir la vulnerabilidad y fortalecer la adaptación de la población, los ecosistemas y los sistemas productivos ante los efectos del cambio climático (México ante el cambio climático, 2021). Sin embargo, los programas de acciones sobre la variabilidad climática, vulnerabilidad y emisiones de gases de efecto invernadero, no son una política permanente idealmente, la política de estado debe enfocarse a proponer estrategias de mitigación y adaptación, sobre la relación costo-beneficio y en promover acciones hacia la sustentabilidad.

La implementación de políticas estratégicas de alcance regional deriva en mejores resultados en términos de manejo del impacto del cambio climático. El éxito podría atribuirse a la necesaria coordinación interinstitucional en los tres niveles de Gobierno. El ámbito de competencia regional incluiría políticas compartidas sobre: 1) calidad del aire; 2) sistemas de transporte (aéreo, marítimo y terrestre); 3) de apoyos fiscales, como por ejemplo préstamos, exenciones en el pago de impuestos, otorgamiento de subsidios a la producción, y precios de garantía; 4) investigación y educación; 5) materiales de construcción; y 6) conservación y aprovechamiento del recurso hídrico (superficial y subterráneo). El punto toral es promover una política de estado enfocada hacia el logro de objetivos comunes en beneficio de la población.

La población debe tener una participación destacada en el delineamiento de políticas regionales, por el conocimiento que tienen del territorio. Es evidente que los impactos del cambio climático son mayores en los sectores más vulnerables, lo que hace necesaria la aplicación de una estrategia política integral e incluyente. Los sistemas de alerta temprana son la herramienta ideal para anunciar la proximidad de eventos meteorológicos extremos (helada, huracán, granizada, chubasco, sequia) y que sean comunicados a través del Tecnologías de Información y Comunicación (TICs), la condición de sequía en el mundo es permanente desde el siglo pasado y su severidad está regionalizada. 
En este contexto, la oferta institucional del INIFAP a través del Laboratorio Nacional de Modelaje y Sensores Remotos, busca reducir el periodo entre la toma de decisiones y su impacto en el corto, mediano y largo plazo. Incluye una serie de productos y servicios web, relacionados con la caracterización del clima y la aplicación de modelos de pronóstico, de alcance regional y nacional. Por ejemplo: el pronóstico numérico de corto plazo, que es una solución reescalada a $8 \mathrm{~km}$, se actualiza cada $24 \mathrm{~h}$, cubre un periodo de cinco días y se presenta en modo diario y horario.

Este producto lo componen 17 variables meteorológicas de: temperatura del aire (mínima, promedio y máxima), temperatura potencial equivalente, temperatura de punto de rocío, temperatura del suelo (0-10 y 10-40 cm), lluvia acumulada, entre otras. Para más información se consulta y descarga las imágenes ingresar a las ligas. (https://clima.inifap.gob.mx/lnmysr/ pronostico/pronosticodiario y https://clima.inifap.gob.mx/lnmysr/pronostico/pronosticohorario).

\section{Radiografía del cambio climático en México}

La frontera espacial del capital natural de México está bajo presión constante debido al crecimiento de los núcleos de población y a la variabilidad climática. Como resultado de ésta, se ha incrementado el área de los ecosistemas de transición. En México el principal ciclo de producción es el primavera-verano y alcanza a cubrir $86 \%$ de la superficie cultivada. Las alteraciones al sector primario que mejor se asocian al cambio climático en México se resumen en la Figura 2.

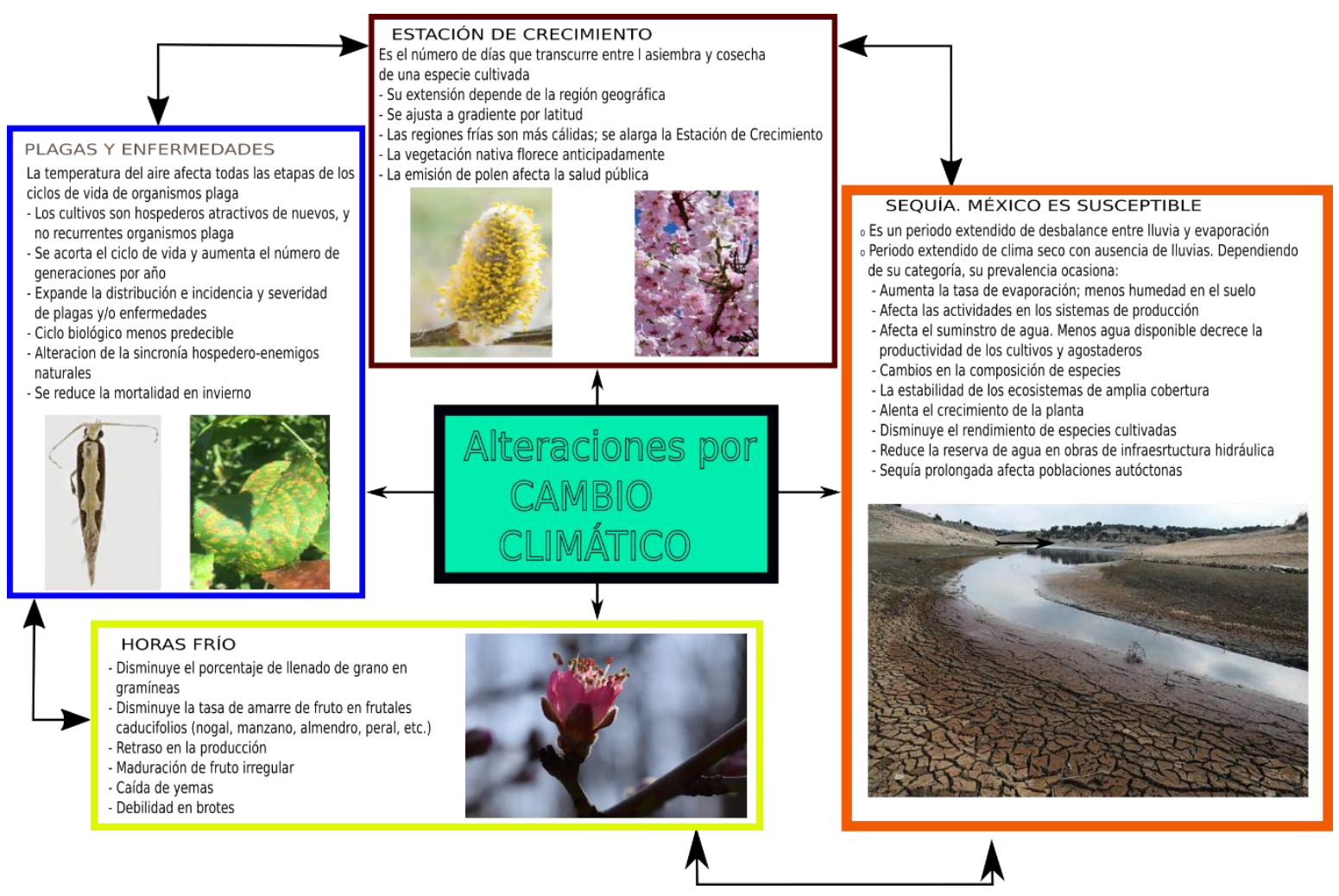

Figura 2. Alteraciones al sector primario atribuidas al cambio climático. Plagas y enfermedades, estación de crecimiento, sequía y horas frío. 
En los ecosistemas abiertos, la principal amenaza es la desestabilización de las cadenas tróficas, de la ganadería extensiva y la modificación de las rutas de vuelo de especies migratorias; la ganadería extensiva en México cubre un aproximado de 109.8 M de ha. La longitud de la estación de crecimiento es contrastante. Está fuertemente influenciada por: temperatura del aire, número de días con heladas, disponibilidad de agua, y el periodo de insolación. Se ha documentado que en la región occidente de México se ha extendido hasta en 2.2 días por década desde 1991, mientras que en la región este la variación es de un día para el mismo periodo. Un aumento en el número de días de la estación de crecimiento resulta en diversificación de cultivos, pero también en la invasión de malas hierbas y un aumento en la demanda de agua.

La condición de sequía en México ha sido una constante de los últimos años. El INIFAP, a través del Laboratorio Nacional de Modelaje y Sensores Remotos, ha implementado la técnica de regresión logística para asignar el valor de clase al tipo de sequía esperado. El pronóstico de sequía resulta del análisis asociativo entre la base de datos categórica del Monitor de Sequía de América del Norte y del acumulado mensual de lluvia obtenido de la serie de tiempo del grupo de amenazas climáticas por precipitación infrarroja con datos de estaciones (CHIRPS) por sus siglas en inglés.

Los mapas se pueden consultar en el portal web institucional https://clima.inifap.gob.mx/lnmysr/ pronostico/sequía. Los periodos de sequía y su intensidad están asociadas a la condición El Niño. El Niño, que es la condición cálida en la temperatura superficial del mar, ocasiona sequías principalmente en estados del sureste de México como Oaxaca, Guerrero y Chiapas, mientras que la condición opuesta, de temperatura fría, también llamada La Niña, corresponde con periodos intensos de sequía en el norte de México. Una caracterización categórica por condición de sequía es la base para delinear programas de uso eficiente del agua en el sector agrícola, industrial y urbano.

\section{La caracterización del espacio geográfico en México}

Ante la evidencia notable del cambio climático y su regionalización, el espacio geográfico puede caracterizarse con base en tres bases de datos mensuales: la cantidad de lluvia acumulada esperada (Figura 3), la anomalía de precipitación esperada con respecto a la climatología base (Figura 4) y la probabilidad de ocurrencia de sequía (Figura 5).

De la Figura 3, el mapa es un producto en proceso de validación. Resulta de implementar una arquitectura de análisis de datos para series de tiempo usando MA. La serie histórica se basa en el volumen acumulado de lluvia mensual obtenida de CHIRPS, a escala nacional. En la Figura 4 se muestra la anomalía de precipitación con respecto a la climatología base.

De la Figura 4, se identifican zonas críticas donde se espera ocurra un déficit en el volumen de lluvia precipitada. Esta regionalización de la condición de sequia podría dirigir el conjunto de acciones de mitigación como un componente de la política pública. La serie de tiempo está pronosticada comparando el escenario de lluvia mensual acumulada (Figura 3) contra la climatología base 2010 publicada por CONAGUA. En la Figura 5 se muestra el pronóstico de sequía. 


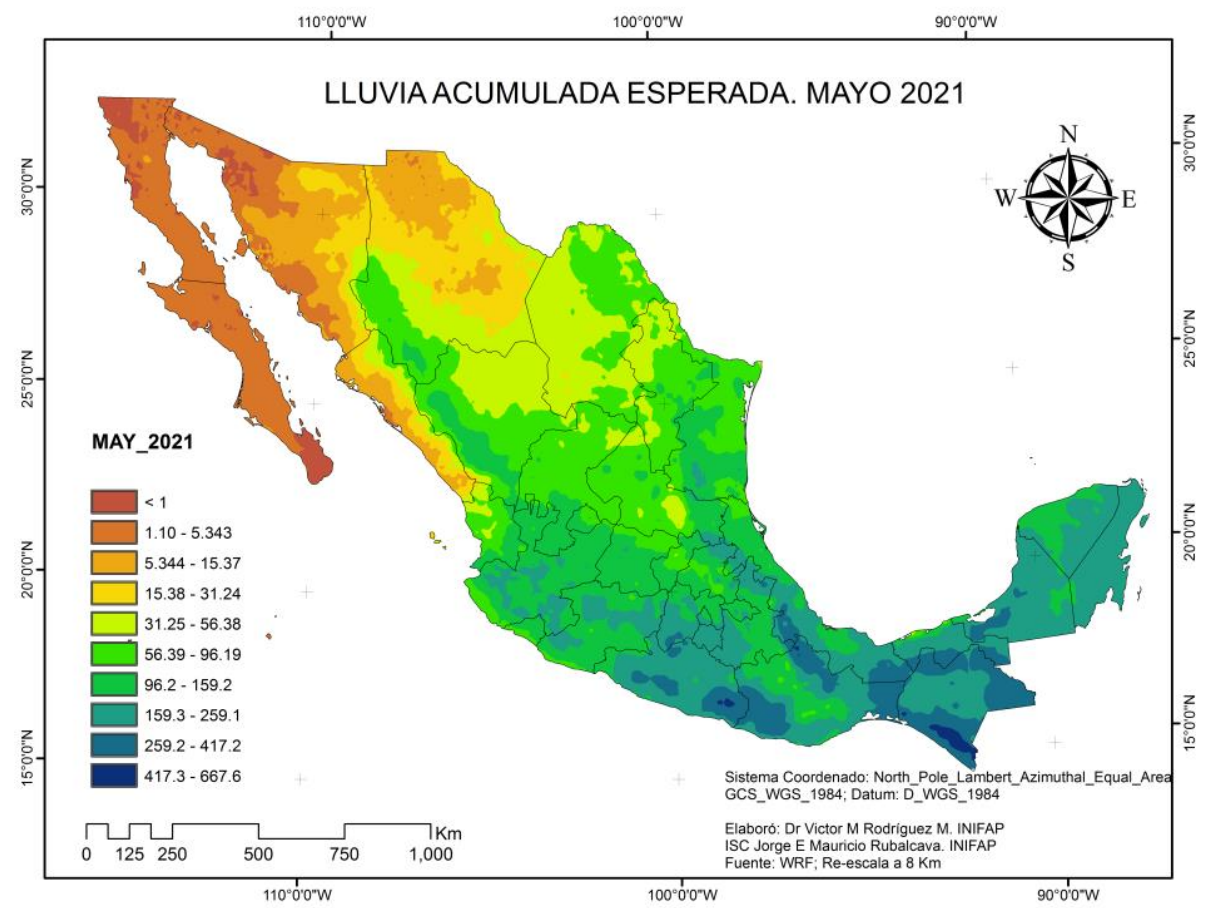

Figura 3. Distribución nacional de lluvia mensual acumulada esperada para el mes de mayo de 2021.

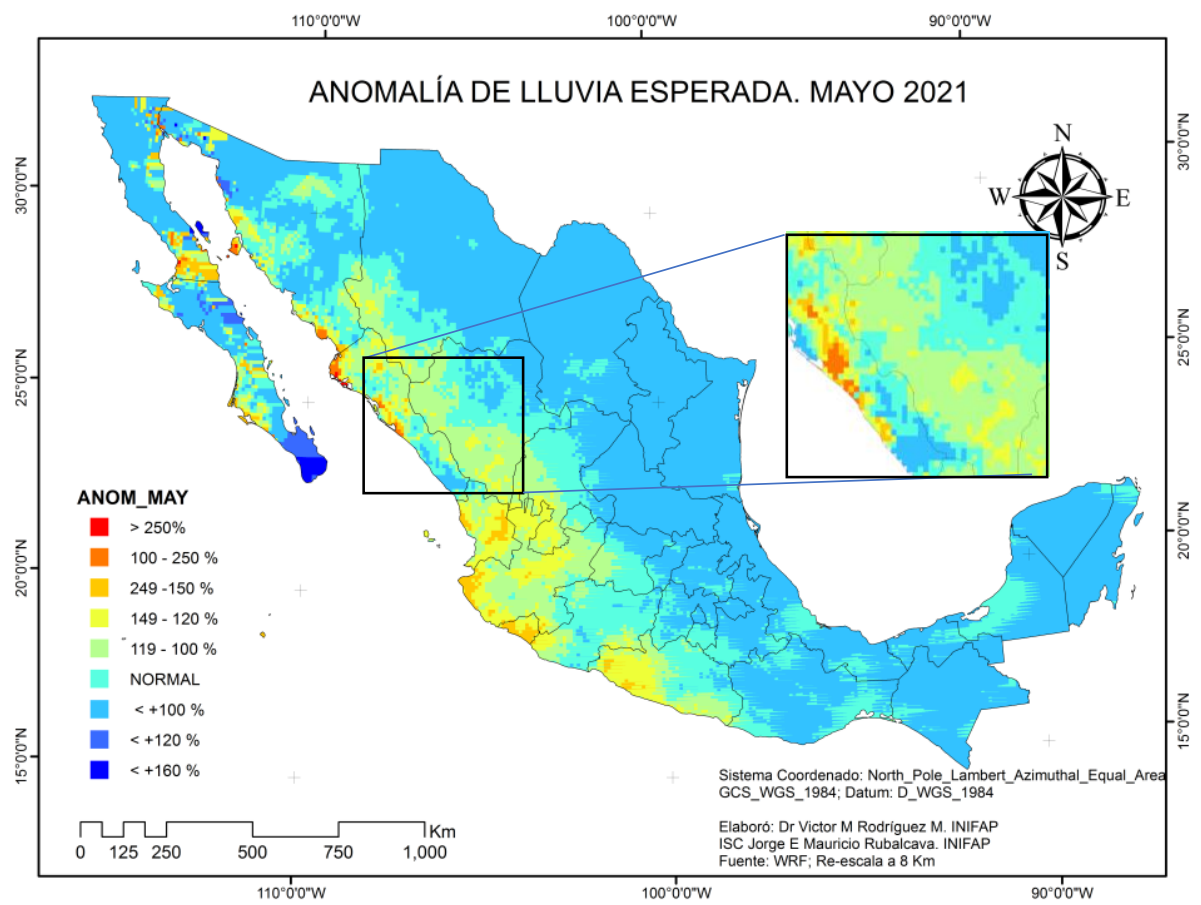

Figura 4. Pronóstico mensual de anomalía de lluvia acumulada (\%). Mayo 2021. 


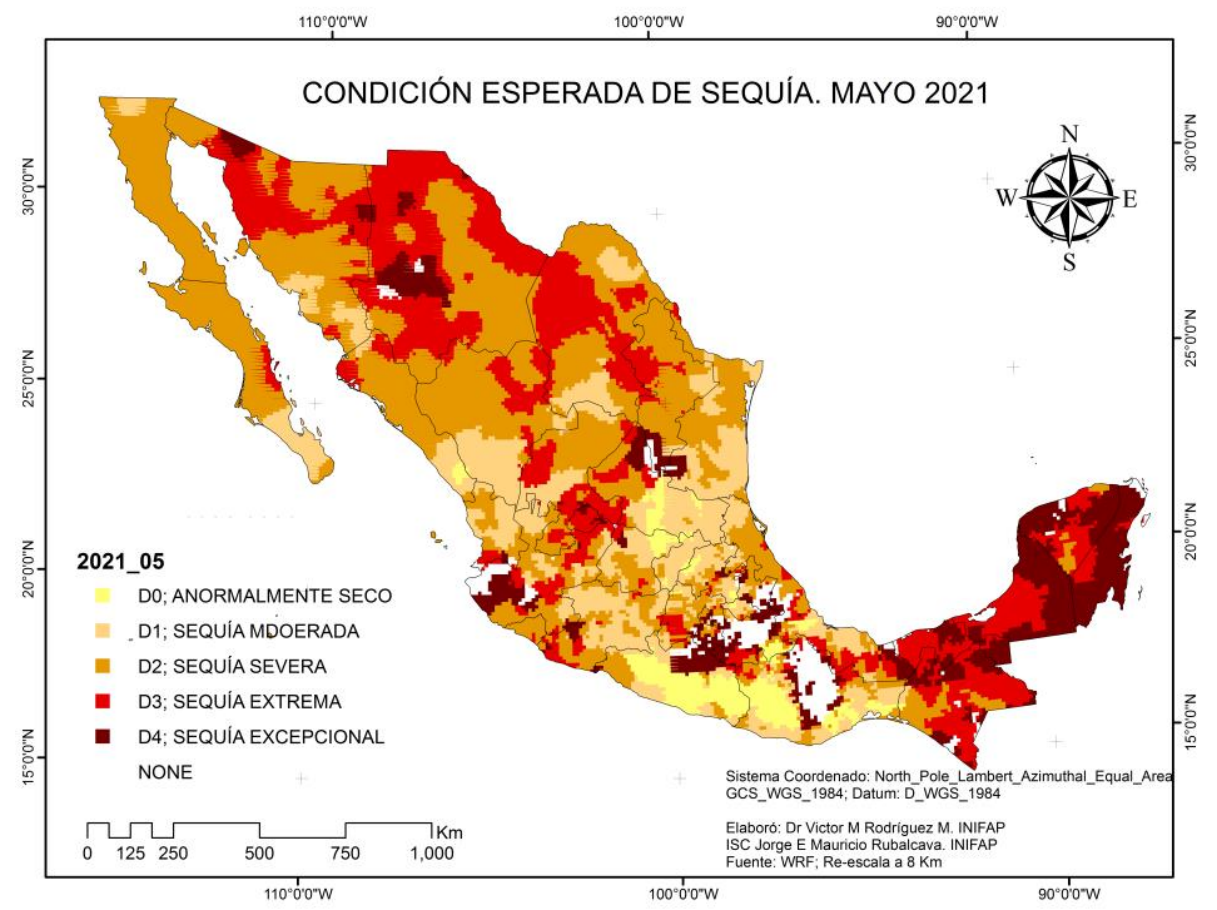

Figura 5. Condición de sequía esperada para México. Mayo de 2021.

A través de la técnica de análisis por regresión logística (ocurre/no ocurre), Se obtuvo el pronóstico mensual de la condición de sequía a escala país. Los mapas obtenidos tienen una solución individual a escala de pixel $(1 \mathrm{~km})$.

\section{El recurso agua en México}

La disponibilidad de agua tiene un componente geográfico. La región norte tiene menos agua disponible, concentra el mayor número de habitantes y económicamente son los estados que más aportan al producto interno bruto (PIB) el $77 \%$ de la población vive en zonas que producen $87 \%$ del PIB de México, pero donde solo se dispone de 31\% del agua. La región Sur es donde hay mayor agua disponible, menos habitantes y es la que menos aporta al PIB (CONAGUA, 2018), aquí se encuentra $69 \%$ de los recursos hídricos de México, vive $23 \%$ de la población y producen $13 \%$ de la riqueza nacional.

Las regiones agrícolas compactas concentran el recurso agua y están destinadas a los agronegocios (Garatuza-Payan et al., 2011), mientras que la demanda de agua por las ciudades aumenta en la misma proporción al número de habitantes. El aumento en la demanda de agua es una amenaza para la sustentabilidad del ecosistema por la sobreexplotación de los acuíferos y el cambio de uso del suelo. Se destaca la minería y la agroindustria que acaparan el agua (Oswald-Spring, 2014).

En este manuscrito se describieron una serie de indicadores numéricos que soportan que México es vulnerable a los efectos del cambio climático. Se destacó la propuesta institucional de INIFAP que propone soluciones a este problema complejo, a través del uso de técnicas efectivas para el manejo de grandes bases de datos y para la generación de pronósticos numéricos y su comunicación 
a través de servicios web. La propuesta resalta los efectos de la variabilidad climática en el sector primario y cómo a través de la ciencia de datos, se obtienen productos temáticos que conjuntan las sinergias entre bases de datos de diseño específico, TICs y modelos de pronóstico de datos.

Las herramientas de MA pueden jugar un papel fundamental para identificar áreas de oportunidad sobre tendencias locales y regionales en los indicadores de impacto del cambio climático y en el delineado de programas de mitigación que integren modelos de respuesta heterogénea y de exploración sobre la interacción de factores ambientales y sociales.

Se puede inferir que los factores climáticos pronosticados de lluvia acumulada, anomalía de lluvia y de condición de sequía, no cubran por completo las expectativas de planeación estratégica como para dirigir una política de estado; sin embargo, son producto de la integración de datos y que prometen ser referentes dada la tendencia a la adopción de sistemas de cómputo en la nube.

Mitigar los impactos del cambio climático requiere de coordinación política efectiva y de una agenda común. Las grandes bases de datos y la sistematización de procesos son el ensamble más efectivo para armonizar el conocimiento científico y dirigir las investigaciones futuras. El modelo propuesto es un castillo del conocimiento (Mauser et al., 2013) que contempla tres etapas: a) codiseño de la investigación; b) coproducción del conocimiento; 3) codiseminación de los resultados. Se codiseña una agenda o programa de investigación común de alcance local, regional y nacional, con la participación de investigadores, tomadores de decisiones y personajes connotados de la población. Los resultados son traducidos a un lenguaje entendible por todos los participantes y se comunica a través de servicios web, talleres virtuales o presenciales y publicaciones técnicas.

\section{Conclusiones}

Es necesario destacar la obligatoriedad de actualizar el marco legal que regule los procesos de seguimiento y dirección de la política social sobre los impactos del cambio climático en México.

\section{Literatura citada}

Almgren, K.; Alshahrani, S. and Lee, J. 2019. Weather data analysis using hadoop to mitigate event planning disasters. https://scholarworks.bridgeport.edu/xmlui/handle/123456789/1105.

Bauer, A; Feichtinger, J. and Steurer, R. 2012. The governance of climate change adaptation in 10 OECD countries: challenges and approaches. J. Environ. Policy Plan 14(12):279-304. https://doi.org/10.1080/1523908X.2012.707406.

CIMA. 2020. Centro de Información de Mercados Agroalimentarios. Reporte del mercado de maíz. https://www.cima.aserca.gob.mx/work/models/cima/pdf/cadena/2020/reporte_mercado_ maiz_200120.

Cherif, R.; Hasanov, F. y Husain, A. 2017. El fin de la era del petróleo: es sólo cuestión de tiempo, FMI Blog, FMN. https://blog-dialogoafondo.imf.org/?p=8291.

CONAGUA. 2018. Estadísticas del agua en México. CONAGUA. Sistema Nacional de Información. Disponible en: http://sina.conagua.gob.mx/publicaciones/EAM_2018.pdf.

Del-Río, J.; Rosales, M.; Ortega, V. y Maya, S. 2016. Análisis de la reforma energética, serie: reformas estructurales: avances y desafíos. Instituto Belisario Domínguez. Senado de la República. número 6. http://bibliodigitalibd.senado.gob.mx/bitstream/handle/123456789/ 3404 / energetica.pdf? sequence $=1 \&$ isAllowed $=\mathrm{y}$. 
Faghmous, J. H. and Kumar, V. 2014. A big data guide to understanding climate change: the case for theory-guided data science. Big Data 2:155-163. doi:10.1089/big.2014.0026.

Fellmann, T. 2012. The assessment of climate change-related vulnerability in the agricultural sector: reviewing conceptual frameworks. In: building resilience for adaptation to climate change in the agriculture sector. FAO/OECD Workshop, Rome, Italy.

Ford, J. D.; Tilleard, S. E.; Berrang-Ford, L.; Araos, M.; Biesbroek, R. and Lesnikowski, A. C. 2016. Opinion: big data has big potential for applications to climate change adaptation. Proc. Natl. Acad. Sci. USA. 113:10729-10732. doi: 10.1073/pnas.1614023113.

Groombridge, B. and Jenkin, M. D. 2002. World Atlas of biodiversity: earth's living resources in the 21st Century. UNEP-WCMC, Cambridge.

Garatuza-Payán, J.; Rodríguez, J. C. and Watts, C. J. 2011. Environmental monitoring and crop water demand. In: Oswald-Spring, Ú. (Ed.). Water resources in Mexico: scarcity, degradation, stress, conflicts, management, and policy. Springer, Berlin. 101-110 p.

Hao, K. 2019. Here are 10 ways AI could help fight climate change. MIT technology review. https://www.technologyreview.com/2019/06/20/134864/ai-climate-change-machinelearning/.

Hua-Dong, G.; Li, Z. and Lan-Wei, Z. 2015. Earth observation big data for climate change research, Adv. Climate Change Res. 6(2):108-117.

Impacto Social Consultores. Panorama. 2021 de energías renovables en México. http://www.impactosocialconsultores.com/blog/2020/5/11/panorama-2020-de-energasrenovables.

Lieverman, D. E. 1999. Vulnerability and adaptation to drought in Mexico. Natural Res. J. 39(1):99-115.

México ante el Cambio Climático. 2021. Información sobre la implementación de la política climática subnacional https://cambioclimatico.gob.mx/informacion-sobre-la-politicaclimatica-subnacional/.

Manogaran, G. and Lopez, D. 2018. Spatial cumulative sum algorithm with big data analytics for climate change detection. Comput. Electr. Eng. 65:207-221. doi: 10.1016/j.compeleceng. 2017.04.006.

Metcalfe, S. E. 1987. Historical data and climate change in Mexico-A Review. Geographical J. 153(2):211-222.

Mauser, W.; Klepper, G.; Rice, M.; Schmalzbauer, B. S.; Hackman, H.; Leemans, R. and Moore, H. 2013. Transdisciplinary global change research: the co-creation of knowledge for sustainability. Curr. Opin. Environ. Sustain. 5(3-4):420-431. Doi:10.1016/ j.cosust.2013.07.001.

Oury, D. T. M. and Singh, A. 2018. Data analysis of weather data using hadoop technology. Smart Computing and Informatics. Springer: Singapore. 723- 730 pp.

Oswald-Spring, Ú. 2014. Water security and national water law in Mexico. Earth perspectives. 1(7):1-15. http://www.earth-perspectives.com/1/1/7.

Oswald-Spring, U. y Brauch, H. G. 2009b. Reconceptualizar la seguridad en el siglo XXI. Centro de Ciencias de la Atmósfera-Centro de Investigaciones Interdisciplinarias en Ciencias y Humanidades-Centro Regional de Investigaciones Multidisciplinarias-Universidad Nacional Autónoma de México (UNAM). Senado de la República LX Legislatura, Cuernavaca, Morelos, México AFES-Press. 887 p. 
Senado de la República. 2003. Dictamen de las comisiones unidas de puntos constitucionales; de energía y estudios legislativos, primera, con proyecto de decreto por el que se reforman y adicionan diversas disposiciones de la constitución política de los Estados Unidos Mexicanos en materia de energía, Senado de la República. http://sil.gobernacion.gob.mx/ archivos/documentos/2013/12/asun_3054419_20131210_1386743657.

SENER. 2019. Secretaría de Energía. Balance nacional de energía 2018. Subsecretaría de Planeación y Transición Energética. Dirección General de Planeación e Información Energéticas. Ciudad de México. 